\title{
Associated production of single top and Higgs at the LHC in the littlest Higgs model with $\mathrm{T}$-parity
}

\author{
Bingfang Yang, ${ }^{a, b}$ Jinzhong $\mathbf{H a n}^{c}$ and Ning Liu ${ }^{a}$ \\ ${ }^{a}$ College of Physics \& Electronic Engineering, Henan Normal University, \\ Xinxiang, 453007 China \\ ${ }^{b}$ School of Materials Science and Engineering, Henan Polytechnic University, \\ Jiaozuo, 454000 China \\ ${ }^{c}$ Department of Physics and Electronic Engineering, Zhoukou Normal University, \\ Zhoukou, 466001 China \\ E-mail: yangbingfang@htu.edu.cn, hanjinzhong@zknu.edu.cn, \\ wlln@mail.ustc.edu.cn
}

ABSTRACT: In the littlest Higgs model with T-parity (LHT), we study the $t$-channel single top production in association with a Higgs boson at 8 and $14 \mathrm{TeV}$ LHC. We find that the cross section can be enhanced obviously in this model compared to the Standard Model. By performing a simple parton-level simulation through $p p \rightarrow t\left(\rightarrow \ell^{+} \nu b\right) h(\rightarrow b \bar{b}) j$ at $14 \mathrm{TeV}$ LHC, we find that the observability of the signal is promising in the favorable parameter space.

Keywords: Monte Carlo Simulations, Hadronic Colliders

ARXIV EPRINT: 1412.2927 


\section{Contents}

1 Introduction 1

2 A brief review of the LHT model 2

3 Numerical results and discussions 3

$\begin{array}{lll}4 & \text { Summary } & 8\end{array}$

\section{Introduction}

On the 4th of July 2012, the ATLAS and CMS collaborations at the Large Hadron Collider (LHC) have discovered a Higgs-like resonance about $125 \mathrm{GeV}[1,2]$. With current data, all properties of the discovered Higgs boson turn out to be in rough agreement with expectations of the Standard Model (SM) [3-6]. Due to the large experimental uncertainties, there remains a plenty of room for new physics in Higgs sector $[7,8]$. In order to ultimately establish its nature, a precise measurement of the Higgs couplings is essential and this task will be performed in the next phase of the LHC and future Higgs factory.

The Yukawa couplings play an important role in probing the new physics since they are sensitive to new flavor dynamics. In view of the large mass, the top quark owns the strongest Yukawa coupling so that it is an appropriate probe for the electroweak symmetry breaking (EWSB) mechanism and new physics [9-11]. As a direct probe of the top Yukawa coupling, the production of a top pair associated with a Higgs boson (t $t \bar{t} h$ production) is a golden channel and has received great attention by the experimenters $[12,13]$ and theorists [14-18]. However, the information on the relative sign between the top Yukawa coupling and Higgs coupling to gauge bosons will still be lacking. In this respect, the production of a single top quark associated with a Higgs boson (thj production) can bring a rather unique possibility [19-22]. The $p p \rightarrow t h j$ production process can be divided into three different modes characterised by the virtuality of the $W$ boson [23]: (i) $t$-channel, where the $W$ is spacelike; (ii) $s$-channel, where the $W$ is timelike; (iii) $W$-associated, where there is emission of a real $W$ boson. Besides, the anomalous $p p \rightarrow t h j$ production process can be induced by the top-Higgs flavor changing neutral current (FCNC) interactions [24-34].

The littlest Higgs model with T-parity (LHT) [35-38] was proposed as a possible solution to the hierarchy problem and so far remains a popular candidate of new physics. The LHT model predicts new gauge bosons, scalars, mirror fermions and top partner, where the T-even top partner $T_{+}$can contribute to the $p p \rightarrow t h j$ process. Furthermore, some Higgs couplings are modified at the high order and this effect can also influence the process $p p \rightarrow t h j$. By performing the detailed analysis on the process $p p \rightarrow t h j$ may 
provide a good opportunity to probe the LHT signal. At the LHC, the $t$-channel process dominates amongst these production modes and the related work has been studied in the littlest Higgs model [39]. In this work, we focus on $t$-channel process and investigate the observability of $p p \rightarrow t h j$ with sequent decays $t \rightarrow \ell^{+} \nu b$ and $h \rightarrow b \bar{b}$ at $14 \mathrm{TeV}$ LHC in the LHT model.

The paper is organized as follows. In section 2 we give a brief review of the LHT model related to our work. In section 3 we calculate the $t$-channel process of $p p \rightarrow t h j$ at the LHC and explore the observability by performing a parton-level simulation. Finally, we give a summary in section 4 .

\section{A brief review of the LHT model}

The LHT model was based on a non-linear $\sigma$ model describing an $\mathrm{SU}(5) / \mathrm{SO}(5)$ symmetry breaking, with the global group $\mathrm{SU}(5)$ being spontaneously broken into $\mathrm{SO}(5)$ by a $5 \times 5$ symmetric tensor at the scale $f \sim \mathcal{O}(\mathrm{TeV})$.

In the top Yukawa Sector, in order to cancel the large radiative correction to Higgs mass parameter induced by top quark, an additional top partner $T_{+}$is introduced, which is even under T-parity and transforms as a singlet under $\mathrm{SU}(2)_{L}$. The implementation of T-parity requires a T-odd mirror partner $T_{-}$. For the top Yukawa interaction, one can write down the following SU(5) and T-parity invariant Lagrangian [35-38]:

$$
\begin{aligned}
\mathcal{L}_{t}= & -\frac{\lambda_{1} f}{2 \sqrt{2}} \epsilon_{i j k} \epsilon_{x y}\left[\left(\bar{Q}_{1}\right)_{i} \Sigma_{j x} \Sigma_{k y}-\left(\bar{Q}_{2} \Sigma_{0}\right)_{i} \tilde{\Sigma}_{j x} \tilde{\Sigma}_{k y}\right] u_{R_{+}} \\
& -\lambda_{2} f\left(\bar{U}_{L_{1}} U_{R_{1}}+\bar{U}_{L_{2}} U_{R_{2}}\right)+\text { h.c. }
\end{aligned}
$$

where $\epsilon_{i j k}$ and $\epsilon_{x y}$ are antisymmetric tensors, and $i, j$ and $k$ run over 1,2,3 and $x$ and $y$ over $4,5 . u_{R_{+}}$and $U_{R_{i}}(i=1,2)$ are $\mathrm{SU}(2)$ singlets.

The heavy quark $T_{+}$mix with the SM top-quark and leads to a modification of the top quark couplings relatively to the SM. The mixing can be parameterized by dimensionless ratio $R=\lambda_{1} / \lambda_{2}$, where $\lambda_{1}$ and $\lambda_{2}$ are two dimensionless top quark Yukawa couplings. This mixing parameter can also be used by $x_{L}$ with

$$
x_{L}=\frac{R^{2}}{1+R^{2}} .
$$

Their masses up to $\mathcal{O}\left(v^{2} / f^{2}\right)$ are given by

$$
\begin{aligned}
m_{t} & =\lambda_{2} \sqrt{x_{L}} v\left[1+\frac{v^{2}}{f^{2}}\left(-\frac{1}{3}+\frac{1}{2} x_{L}\left(1-x_{L}\right)\right)\right], \\
m_{T_{+}} & =\frac{f}{v} \frac{m_{t}}{\sqrt{x_{L}\left(1-x_{L}\right)}}\left[1+\frac{v^{2}}{f^{2}}\left(\frac{1}{3}-x_{L}\left(1-x_{L}\right)\right)\right], \\
m_{T_{-}} & =\frac{f}{v} \frac{m_{t}}{\sqrt{x_{L}}}\left[1+\frac{v^{2}}{f^{2}}\left(\frac{1}{3}-\frac{1}{2} x_{L}\left(1-x_{L}\right)\right)\right],
\end{aligned}
$$




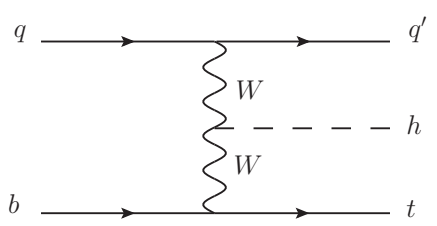

$(a)$

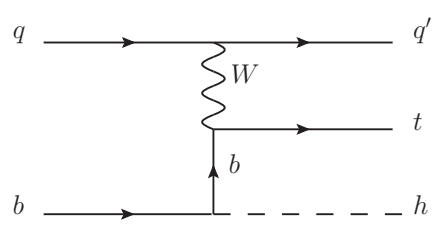

(b)

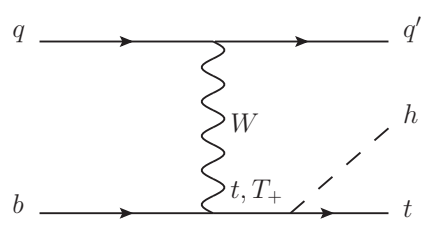

(c)

Figure 1. Feynman diagrams for $p p \rightarrow t h j$ in the LHT model at the tree level.

where $v=v_{\mathrm{SM}}\left(1+\frac{1}{12} \frac{v_{\mathrm{SM}}^{2}}{f^{2}}\right)$ and $v_{\mathrm{SM}}=246 \mathrm{GeV}$ is the SM Higgs VEV. Some typical Higgs couplings involved in our calculations are given by

$$
\begin{aligned}
& V_{H b \bar{b}}=-\frac{m_{b}}{v}\left(1-\frac{1}{6} \frac{v^{2}}{f^{2}}\right), \\
& V_{H W_{\mu} W_{\nu}}=\frac{2 m_{W}^{2}}{v}\left(1-\frac{1}{6} \frac{v^{2}}{f^{2}}\right) g_{\mu \nu}, \\
& V_{W_{\mu} \bar{t} b}=\frac{V_{t b}}{\sqrt{2}} g \gamma_{\mu}\left(1-\frac{x_{L}^{2}}{2} \frac{v^{2}}{f^{2}}\right) P_{L}, \\
& V_{W_{\mu} \bar{T}_{+} b}=\frac{V_{t b}}{\sqrt{2}} g \gamma_{\mu} x_{L} \frac{v}{f} P_{L}, \\
& V_{h t \bar{t}}=-\frac{m_{t}}{v}\left[1+\frac{v^{2}}{f^{2}}\left(-\frac{2}{3}+x_{L}-x_{L}^{2}\right)\right], \\
& V_{h t \bar{T}_{+}}=-m_{t}\left[\frac{\left(1-x_{L}\right)}{f} P_{R}-\frac{\sqrt{x_{L}}}{v \sqrt{1-x_{L}}} P_{L}\right] \text {, }
\end{aligned}
$$

where $P_{L}=\frac{1-\gamma_{5}}{2}$ and $P_{R}=\frac{1+\gamma_{5}}{2}$ are chirality projection operators. The Higgs coupling with down-type quarks have two different cases [40], namely case A and case B.

\section{Numerical results and discussions}

In the LHT model, the lowest-order Feynman diagrams of the process $p p \rightarrow t h j(j \neq b)$ are shown in figure 1 . We can see that the T-even heavy quark $T_{+}$contributes this process through the figure 1(c). In our calculations, the conjugate process $p p \rightarrow \bar{t} h j$ has been considered, unless otherwise noted.

We compute the cross sections using the MadGraph 5 [41] (and checked against those obtained by CalcHEP 3.6.22 [42, 43]), where the CTEQ6L [44] is used as the parton distribution function and the renormalization scale $\mu_{R}$ and factorization scale $\mu_{F}$ is set to be $\mu_{R}=\mu_{F}=m_{t}+m_{h}$. The relevant SM input parameters are taken as follows [45]:

$$
\begin{aligned}
m_{t} & =173.07 \mathrm{GeV}, & m_{Z} & =91.1876 \mathrm{GeV}, & \alpha\left(m_{Z}\right) & =1 / 128, \\
\sin ^{2} \theta_{W} & =0.231, & m_{h} & =125 \mathrm{GeV}, & \alpha_{s}\left(m_{Z}\right) & =0.1185 .
\end{aligned}
$$

The relative correction of the cross section can be defined as

$$
\delta \sigma / \sigma=\frac{\sigma-\sigma_{\mathrm{SM}}}{\sigma_{\mathrm{SM}}} .
$$




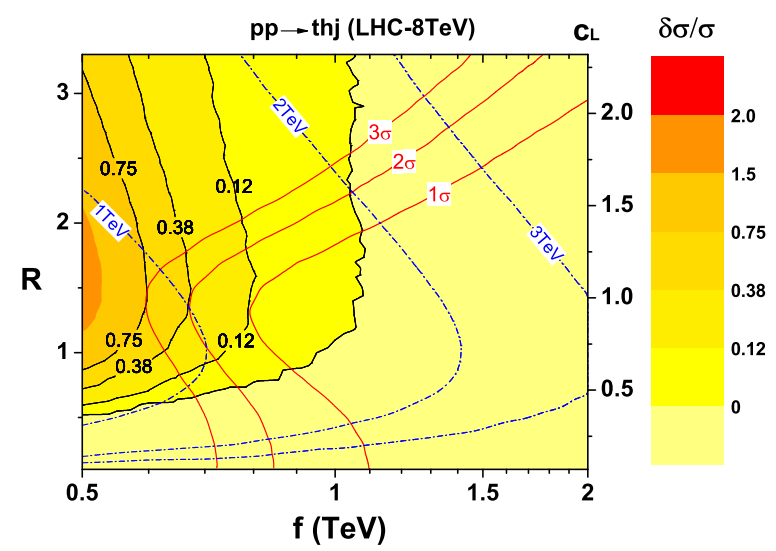

Figure 2. The relative corrections $(\delta \sigma / \sigma)_{t h j}$ at $8 \mathrm{TeV}$ LHC in the LHT model. The red solid lines respectively represent the $1 \sigma, 2 \sigma$ and $3 \sigma$ confidence regions, the blue dash-dot lines respectively represent the $m_{T_{+}}=1 \mathrm{TeV}, 2 \mathrm{TeV}$ and $3 \mathrm{TeV}$.

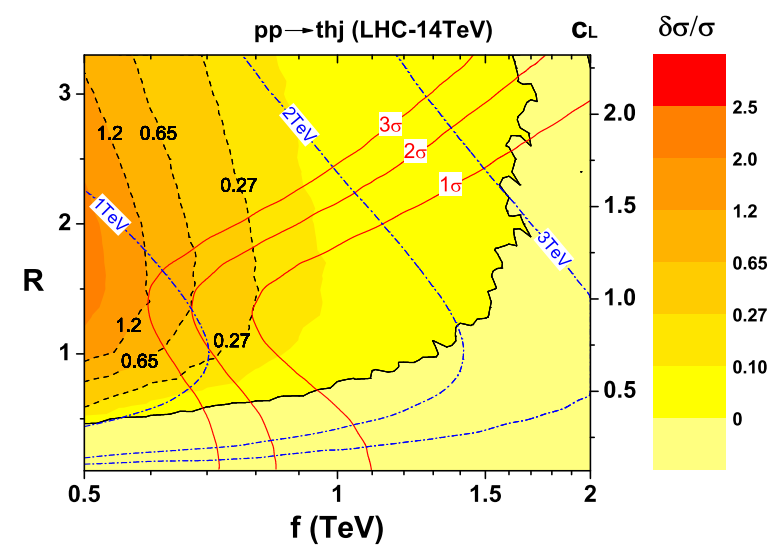

Figure 3. The relative corrections $(\delta \sigma / \sigma)_{t h j}$ at $14 \mathrm{TeV}$ LHC in the LHT model. The red solid lines respectively represent the $1 \sigma, 2 \sigma$ and $3 \sigma$ confidence regions, the blue dash-dot lines respectively represent the $m_{T_{+}}=1 \mathrm{TeV}, 2 \mathrm{TeV}$ and $3 \mathrm{TeV}$.

In our calculations, the leading-order cross sections for the processes $p p \rightarrow t h j$ in the SM are taken as $\sigma_{\mathrm{SM}}^{8 \mathrm{TeV}}=16.4 \mathrm{fb}$ and $\sigma_{\mathrm{SM}}^{14 \mathrm{TeV}}=80.4 \mathrm{fb}$.

Our results show that the features of the process $p p \rightarrow t h j$ are very similar for the case A and case B, so here we focus on the case A scenario. The LHT parameters related to our calculations are the scale $f$ and the ratio $R$. Considering the consistent constraints in refs. [46-48], we require the scale $f$ and the ratio $R$ to vary in the range $500 \mathrm{GeV} \leq f \leq 2000 \mathrm{GeV}$ and $0.1 \leq R \leq 3.3$. Combined with the global fit of the current Higgs data and the electroweak precision observables (EWPO) in ref. [49], the confidence regions (corresponding to $1 \sigma, 2 \sigma$ and $3 \sigma$ ranges for case A) are provided in figures 2,3 . Furthermore, according to refs. $[50,51]$, we can see that the constraints on the LHT parameters from the dark matter observations are weaker than the Higgs data and EWPO at $2 \sigma$ level, which means that the parameter space allowed by the Higgs data and EWPO can also satisfy the dark matter constraints. 
In figure 2 and figure 3 , we show the relative corrections $\delta \sigma / \sigma$ of the processes $p p \rightarrow t h j$ at the 8 and $14 \mathrm{TeV}$ LHC in the LHT model, respectively. From the figure 2 and figure 3 , we can see that the relative corrections $\delta \sigma / \sigma$ of $p p \rightarrow t h j$ at 8 and $14 \mathrm{TeV}$ LHC can be respectively reach $38 \%$ and $65 \%$ at $2 \sigma$ level. We find that these large corrections mainly come from the resonance decay of the heavy quark $T_{+}$in the figure 1(c). Furthermore, we can see that the relative corrections $\delta \sigma / \sigma$ are negative in considerable regions for $8 \mathrm{TeV}$ LHC and non-negligible regions for $14 \mathrm{TeV}$ LHC. The main reasons are as follows:

Due to the small coupling $h b \bar{b}$, the main contribution to the $p p \rightarrow t h j$ comes from figures $1(\mathrm{a}), 1(\mathrm{c})$. If we take no account of the heavy quark $T_{+}$, we can see that the couplings $h W W$ and $h t \bar{t}$ have the opposite sign so that the contributions of figure 1(a) and figure 1(c) cancel each other. According to the eq. (10), we can see that the left-handed part $\left(c_{L}=m_{t} \frac{R}{v}\right)$ of the coupling $h t \bar{T}_{+}$has the same sign as the coupling $h W W$ so that their contributions enhance each other. The same thing happens between the right-handed part $\left(c_{R}=-m_{t} \frac{\left(1-x_{L}\right)}{f}\right)$ of the coupling $h t \bar{T}_{+}$and the coupling $h t \bar{t}$. As a result, the total contribution induced by the top partner $T_{+}$depends on the surplus after the cancelation between $c_{L}$ contribution and $c_{R}$ contribution. One can notice that the left-handed coupling $c_{L}(\propto R)$ is proportional to the ratio $R$ and dominates the contribution from the heavy quark $T_{+}$. Moreover, the Higgs couplings in the LHT model are modified at $\mathcal{O}\left(v^{2} / f^{2}\right)$, which can decrease the $t h j$ cross section. Combining these factors above, we can see that the large relative corrections $\delta \sigma / \sigma$ come from the region that has small $f$, small $m_{T_{+}}$and large $c_{L}$. By contrast, the small or negative relative corrections $\delta \sigma / \sigma$ come from the region that has large $f$, large $m_{T_{+}}$, small $c_{L}$ or the combination of them. Due to the lower centre-of-mass energy, the relative corrections $\delta \sigma / \sigma$ at $8 \mathrm{TeV}$ LHC are suppressed by the large $m_{T_{+}}$more strongly so that the negative $\delta \sigma / \sigma$ regions are larger compared to the case for $14 \mathrm{TeV}$ LHC. Furthermore, for the same ratio $R$, we can see that the relative corrections $\delta \sigma / \sigma$ of $p p \rightarrow t h j$ at 8 and $14 \mathrm{TeV}$ LHC both decrease with the scale $f$ increasing, which means that the LHT effect decouples with the scale $f$ increasing.

In the following calculations, we will perform a simple parton-level simulation and explore the sensitivity of $14 \mathrm{TeV}$ LHC through the channel $p p \rightarrow t\left(\rightarrow \ell^{+} \nu b\right) h(\rightarrow b \bar{b}) j$, the signal is characterised by

$$
1 \text { forward et }+3 b+\ell^{+}+E_{T}
$$

where $j$ denotes the light jets and $\ell=e, \mu$. The most relevant backgrounds can be divided into two classes:

(i) reducible backgrounds, $p p \rightarrow t \bar{t}(\rightarrow \bar{b} \bar{c} s)$ and $p p \rightarrow t \bar{t}(\rightarrow \bar{b} \bar{c} s) j$;

(ii) irreducible backgrounds, $p p \rightarrow t Z(\rightarrow b \bar{b}) j$ and $p p \rightarrow t b \bar{b} j$.

Signal and background events have been generated at the parton level using MadGraph 5, the subsequent simulations are performed by MadAnalysis 5 [52]. To simulate $b$-tagging, we take moderate single $b$-tagging efficiency $\epsilon_{b}=0.6$ for $b$-jets in the final state. We also include charm mistag probability $\epsilon_{c}=0.08$ and light jet mistag probability $\epsilon_{j}=0.004$ in the reducible backgrounds [53, 54]. Follow the analysis on $t \bar{t} h$ signature by 

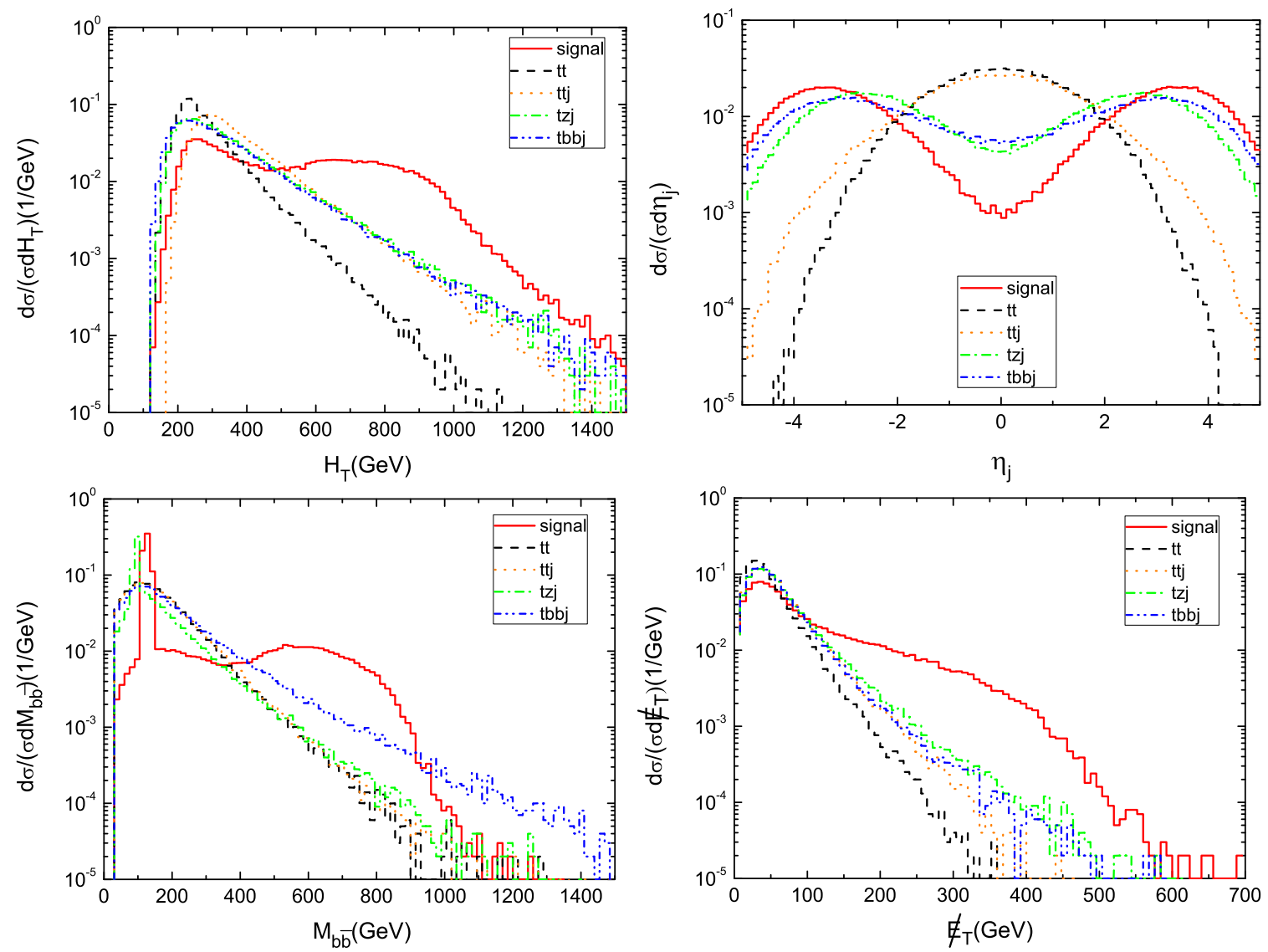

Figure 4. The normalised distributions of $H_{T}, \eta_{j}, M_{b \bar{b}}, E_{T}$ after the basic cuts in the signal and backgrounds at $14 \mathrm{TeV}$ LHC for $f=700 \mathrm{GeV}, R=1.5$.

ATLAS and CMS collaborations $[12,13]$ at the LHC Run-I, we chose the basic cuts as follows:

$$
\begin{aligned}
\Delta R_{i j} & >0.4, & & i, j=b, j \text { or } \ell \\
p_{T}^{b} & >25 \mathrm{GeV}, & & \left|\eta_{b}\right|<2.5 \\
p_{T}^{\ell} & >25 \mathrm{GeV}, & & \left|\eta_{\ell}\right|<2.5 \\
p_{T}^{j} & >25 \mathrm{GeV}, & & \left|\eta_{j}\right|<5 .
\end{aligned}
$$

After basic cuts, the signal is overwhelmed by the backgrounds. In order to reduce the contributions of the backgrounds and enhance the signal contribution, some additional cuts are required and some other kinematic distributions are needed. As an example, we display the normalised distributions of $H_{T}, \eta_{j}, M_{b \bar{b}}, E_{T}$ in the signal and backgrounds at $14 \mathrm{TeV}$ LHC for $f=700 \mathrm{GeV}, R=1.5$ in figure 4 , where $H_{T}\left(=\sum_{\text {hadronic particles }}\left\|\vec{p}_{T}\right\|\right)$ is the total transverse hadronic energy, $\eta_{j}$ is pseudorapidity of the leading jet, $M_{b \bar{b}}$ is the invariant mass of the two $b$-jets from the Higgs boson decay and $E_{T}\left(=\left\|\sum_{\text {visible particles }} \vec{p}_{T}\right\|\right)$ is the missing transverse energy.

Firstly, we can see that there is a bulge in the $H_{T}$ distribution of the signal, which arises from the resonance effect of the top partner $T_{+}$and this effect also appears in some 


\begin{tabular}{|c|c|c|c|c|c|c|c|}
\hline \multirow{3}{*}{ Cuts } & \multicolumn{5}{|c|}{$\sigma(\mathrm{fb})$} & \multirow{3}{*}{$\begin{array}{c}\frac{S}{\sqrt{S+B}} \\
300 \mathrm{fb}^{-1}\end{array}$} & \multirow{3}{*}{$\begin{array}{l}\frac{S}{B} \\
\%\end{array}$} \\
\hline & \multirow{2}{*}{$\begin{array}{c}\text { Signal } \\
t h j \\
\end{array}$} & \multicolumn{4}{|c|}{ Backgrounds } & & \\
\hline & & $t \bar{t}$ & $t \bar{t} j$ & $t Z j$ & $t b \bar{b} j$ & & \\
\hline \multirow{2}{*}{ Basic cuts } & $1.12(1.34)$ & 702.7 & 648.7 & 1.68 & 2.82 & $0.53(0.63)$ & $0.083(0.099)$ \\
\hline & $0.72(0.74)$ & 702.7 & 648.7 & 1.68 & 2.82 & $0.34(0.35)$ & $0.053(0.055)$ \\
\hline$H_{T}>530 \mathrm{GeV}$ & $0.45(0.65)$ & 15.27 & 69.58 & 0.16 & 0.25 & $0.85(1.22)$ & $0.53(0.77)$ \\
\hline$H_{T}>600 \mathrm{GeV}$ & $0.10(0.11)$ & 7.22 & 40.24 & 0.10 & 0.16 & $0.25(0.29)$ & $0.21(0.24)$ \\
\hline \multirow{2}{*}{$\left|\eta_{j}\right|>2$} & $0.36(0.54)$ & 0.6 & 11.13 & 0.074 & 0.13 & $1.78(2.65)$ & $3.0(4.5)$ \\
\hline & $0.07(0.084)$ & 0.23 & 5.98 & 0.042 & 0.076 & $0.48(0.57)$ & $1.1(1.3)$ \\
\hline \multirow{2}{*}{$\left|M_{b \bar{b}}-m_{h}\right|<15 \mathrm{GeV}$} & $0.12(0.18)$ & 0.004 & 0.99 & 0.0029 & 0.0034 & $1.96(2.87)$ & $12(18)$ \\
\hline & $0.023(0.028)$ & 0. & 0.47 & 0.0015 & 0.0019 & $0.57(0.68)$ & $4.9(5.9)$ \\
\hline$E_{T}>100 \mathrm{GeV}$ & $0.078(0.12)$ & 0. & 0.23 & 0.001 & 0.001 & $2.4(3.6)$ & $33.6(53.5)$ \\
\hline$E_{T}>180 \mathrm{GeV}$ & $0.01(0.013)$ & 0. & 0.036 & 0.0002 & 0.0002 & $0.81(1.02)$ & $27.8(36.1)$ \\
\hline
\end{tabular}

Table 1. Cutflow of the cross sections for the signal and backgrounds at $14 \mathrm{TeV}$ LHC on the benchmark points [top-left: $(f=700 \mathrm{GeV}, R=1)$; top-right: $(f=700 \mathrm{GeV}, R=1.5)$; bottom-left: $(f=1000 \mathrm{GeV}, R=1)$; bottom-right: $(f=1000 \mathrm{GeV}, R=1.5)]$. All the conjugate processes of the signal and backgrounds have been included.

other distributions. So we require the events to satisfy $H_{T}>530 \mathrm{GeV}$ to isolate the signal and find all the backgrounds are suppressed effectively. After this cut, the backgrounds are still much larger than the signal, especially the two reducible backgrounds $t \bar{t}$ and $t \bar{t} j$. According to the $\eta_{j}$ distribution, we can see that most events of $t \bar{t}$ and $t \bar{t} j$ have a leading jet in the central region, which differs significantly from the signal, so we apply the cut $\left|\eta_{j}\right|>2$ to further suppress the $t \bar{t}$ and $t \bar{t} j$ backgrounds.

Another effective cut which can suppress the backgrounds is the invariant mass cut on the two $b$-jets from the Higgs boson decay. The $b$ quark from the top decay can be selected with high purity by choosing the smallest invariant masses $M_{b l}$ of each $b$-jet and the lepton among the three combinations [54]. The other two $b$ quarks are then considered the $b$ quarks coming from the Higgs boson decay. We find that the signal peak of $M_{b \bar{b}}$ is more narrow than those of the backgrounds, so we use the cut $\left|M_{b \bar{b}}-m_{h}\right|<15 \mathrm{GeV}$ to enhance the observability of the signal. Besides, we apply the cut $E_{T}>100 \mathrm{GeV}$ to further isolate the signal and find that the $t \bar{t} j$ background is suppressed effectively. After all cuts above, the background is dominated by $t \bar{t} j$ completely due to an extra hard jet with $t \bar{t}$.

For easy reading we summarize the cut-flow cross sections of the signal and backgrounds for $14 \mathrm{TeV}$ LHC in table 1. For comparison, we chose four sets of benchmark points, that is $(f=700 \mathrm{GeV}, R=1),(f=700 \mathrm{GeV}, R=1.5),(f=1000 \mathrm{GeV}, R=1)$ and ( $f=1000 \mathrm{GeV}, R=1.5$ ), they are arranged in the top-left (top-right) and bottom-left (bottom-right) part of one cell, respectively. Due to the resonance effect of the top partner $T_{+}$, the values of the cuts, mainly the $H_{T}$ and $E_{T}$ cuts, need to be changed with the top partner mass in order to better suppress the backgrounds. From the table 2, we can see that the larger $m_{T_{+}}$will correspond to the larger $H_{T}$ and $E_{T}$ cuts. Because of the 


\begin{tabular}{|r|c|c|c|}
\hline \multicolumn{1}{c|}{ Benchmark point } & $m_{T_{+}}(\mathrm{GeV})$ & $H_{T^{-} \text {cut }(\mathrm{GeV})}$ & $E_{T^{-}}$cut $(\mathrm{GeV})$ \\
\hline$(f=700 \mathrm{GeV}, R=1)$ & 993.8 & $>530$ & $>100$ \\
$(f=700 \mathrm{GeV}, R=1.5)$ & 1081.5 & $>530$ & $>100$ \\
\hline$(f=1000 \mathrm{GeV}, R=1)$ & 1412.3 & $>600$ & $>180$ \\
$(f=1000 \mathrm{GeV}, R=1.5)$ & 1533.5 & $>600$ & $>180$ \\
\hline
\end{tabular}

Table 2. The top partner mass $m_{T_{+}}$and the corresponding $H_{T}$ and $E_{T}$ cuts for the four sets of benchmark points.

approximate $m_{T_{+}}$, the same values of $H_{T}\left(\right.$ or $\left.E_{T}\right)$ cuts are taken for the benchmark points $(f=700 \mathrm{GeV}, R=1)$ and $(f=700 \mathrm{GeV}, R=1.5)$ in our simulation, and so are the benchmark points $(f=1000 \mathrm{GeV}, R=1)$ and $(f=1000 \mathrm{GeV}, R=1.5)$.

In order to analyze the observability, we calculate the signal-to-background ratio according to $S / \sqrt{S+B}$ and the systematic significance $S / B$ for the luminosity $\mathcal{L}=300 \mathrm{fb}^{-1}$, where $S$ represents the number of signal events and $B$ represents the number of background events. From table 1, we can see that $S / \sqrt{S+B}$ and $S / B$ are substantially improved by these selected cuts, where the signal-to-background ratio $S / \sqrt{S+B}$ can reach $3.6 \sigma$ and systematic significance $S / B$ can reach $53.5 \%$ for $f=700 \mathrm{GeV}, R=1.5$. Moreover, it's worth noting that the systematic significance $S / B$ is enhanced obviously, which will help to draw the signal from the backgrounds.

\section{Summary}

In the framework of the LHT model, we investigate the $t$-channel process of $p p \rightarrow t h j$ at 8 and $14 \mathrm{TeV}$ LHC. With current constraints, we find that the cross section can be enhanced obviously in some parameter space compared to the SM predictions. We further investigate the observability of $p p \rightarrow t h j$ with decays $t \rightarrow \ell^{+} \nu b$ and $h \rightarrow b \bar{b}$ at $14 \mathrm{TeV} \mathrm{LHC}$ for some benchmark points. By performing a simple parton-level simulation, we find that the observability of the LHT signal is promising at the high-luminosity LHC.

\section{Acknowledgments}

We would like to thank Lei Wu for helpful suggestions. This work was supported by the National Natural Science Foundation of China (NNSFC) under grants Nos. 11405047, 11305049, by the China Postdoctoral Science Foundation under Grant No. 2014M561987 and the Joint Funds of the National Natural Science Foundation of China (U1404113).

Open Access. This article is distributed under the terms of the Creative Commons Attribution License (CC-BY 4.0), which permits any use, distribution and reproduction in any medium, provided the original author(s) and source are credited. 


\section{References}

[1] ATLAS collaboration, Observation of a new particle in the search for the Standard Model Higgs boson with the ATLAS detector at the LHC, Phys. Lett. B 716 (2012) 1 [arXiv:1207.7214] [INSPIRE].

[2] CMS collaboration, Observation of a new boson at a mass of $125 \mathrm{GeV}$ with the CMS experiment at the LHC, Phys. Lett. B 716 (2012) 30 [arXiv:1207.7235] [INSPIRE].

[3] ATLAS collaboration, Measurements of the properties of the Higgs-like boson in the four lepton decay channel with the ATLAS detector using $25 \mathrm{fb}^{-1}$ of proton-proton collision data, ATLAS-CONF-2013-013 (2013) [ATLAS-COM-CONF-2013-018] [INSPIRE].

[4] ATLAS collaboration, Combined coupling measurements of the Higgs-like boson with the ATLAS detector using up to $25 \mathrm{fb}^{-1}$ of proton-proton collision data, ATLAS-CONF-2013-034 (2013) [ATLAS-COM-CONF-2013-035] [INSPIRE].

[5] ATLAS collaboration, Study of the spin of the new boson with up to $25 \mathrm{fb}^{-1}$ of ATLAS data, ATLAS-CONF-2013-040 (2013) [ATLAS-COM-CONF-2013-048] [INSPIRE].

[6] CMS collaboration, Combination of standard model Higgs boson searches and measurements of the properties of the new boson with a mass near $125 \mathrm{GeV}$, CMS-PAS-HIG-13-005 (2013) [INSPIRE].

[7] S. Dawson et al., Working Group Report: Higgs Boson, arXiv:1310.8361 [INSPIRE].

[8] C. Englert et al., Precision Measurements of Higgs Couplings: Implications for New Physics Scales, J. Phys. G 41 (2014) 113001 [arXiv:1403.7191] [InSPIRE].

[9] J.J. Cao, Z.X. Heng, L. Wu and J.M. Yang, Top quark forward-backward asymmetry at the Tevatron: A Comparative study in different new physics models, Phys. Rev. D 81 (2010) 014016 [arXiv:0912.1447] [INSPIRE].

[10] J.J. Cao, L. Wu and J.M. Yang, New physics effects on top quark spin correlation and polarization at the LHC: a comparative study in different models, Phys. Rev. D 83 (2011) 034024 [arXiv: 1011.5564] [INSPIRE].

[11] B.F. Yang and N. Liu, One-loop effects on top pair production in the littlest Higgs model with T-parity at the LHC, Eur. Phys. J. C 73 (2013) 2570 [arXiv:1210.5120] [InSPIRE].

[12] ATLAS collaboration, Search for the Standard Model Higgs boson produced in association with top quarks in proton-proton collisions at $\sqrt{s}=7$ TeV using the ATLAS detector, ATLAS-CONF-2012-135 (2012) [ATLAS-COM-CONF-2012-162] [INSPIRE].

[13] CMS collaboration, Search for Higgs boson production in association with top quark pairs in p p collisions, CMS-PAS-HIG-12-025 (2012) [INSPIRE].

[14] W. Beenakker, S. Dittmaier, M. Krämer, B. Plumper, M. Spira and P.M. Zerwas, Higgs radiation off top quarks at the Tevatron and the LHC, Phys. Rev. Lett. 87 (2001) 201805 [hep-ph/0107081] [INSPIRE].

[15] S. Dawson, L.H. Orr, L. Reina and D. Wackeroth, Associated top quark Higgs boson production at the LHC, Phys. Rev. D 67 (2003) 071503 [hep-ph/0211438] [InSPIRE].

[16] R. Frederix, S. Frixione, V. Hirschi, F. Maltoni, R. Pittau and P. Torrielli, Scalar and pseudoscalar Higgs production in association with a top-antitop pair, Phys. Lett. $\mathbf{B} 701$ (2011) 427 [arXiv:1104.5613] [INSPIRE].

[17] M.V. Garzelli, A. Kardos, C.G. Papadopoulos and Z. Trócsányi, Standard Model Higgs boson production in association with a top anti-top pair at NLO with parton showering, Europhys. Lett. 96 (2011) 11001 [arXiv:1108.0387] [INSPIRE]. 
[18] C. Degrande, J.M. Gerard, C. Grojean, F. Maltoni and G. Servant, Probing Top-Higgs Non-Standard Interactions at the LHC, JHEP 07 (2012) 036 [arXiv:1205.1065] [INSPIRE].

[19] G. Bordes and B. van Eijk, On the associate production of a neutral intermediate mass Higgs boson with a single top quark at the LHC and SSC, Phys. Lett. B 299 (1993) 315 [INSPIRE].

[20] A. Ballestrero and E. Maina, $t \bar{b} H$ production for an intermediate mass Higgs, Phys. Lett. B 299 (1993) 312 [INSPIRE].

[21] W.J. Stirling and D.J. Summers, Production of an intermediate mass Higgs boson in association with a single top quark at LHC and SSC, Phys. Lett. B 283 (1992) 411 [INSPIRE].

[22] J.L. Diaz-Cruz and O.A. Sampayo, Associated production of the Higgs boson with $t \bar{b}$ at hadron colliders, Phys. Lett. B 276 (1992) 211 [INSPIRE].

[23] F. Maltoni, K. Paul, T. Stelzer and S. Willenbrock, Associated production of Higgs and single top at hadron colliders, Phys. Rev. D 64 (2001) 094023 [hep-ph/0106293] [INSPIRE].

[24] M. Farina, C. Grojean, F. Maltoni, E. Salvioni and A. Thamm, Lifting degeneracies in Higgs couplings using single top production in association with a Higgs boson, JHEP 05 (2013) 022 [arXiv: 1211.3736] [INSPIRE].

[25] L. Wu, Enhancing thj Production from Top-Higgs FCNC Couplings, JHEP 02 (2015) 061 [arXiv: 1407.6113] [INSPIRE].

[26] A. Kobakhidze, L. Wu and J. Yue, Anomalous Top-Higgs Couplings and Top Polarisation in Single Top and Higgs Associated Production at the LHC, JHEP 10 (2014) 100 [arXiv: 1406.1961] [INSPIRE].

[27] A. Greljo, J.F. Kamenik and J. Kopp, Disentangling Flavor Violation in the Top-Higgs Sector at the LHC, JHEP 07 (2014) 046 [arXiv:1404.1278] [INSPIRE].

[28] S. Khatibi and M.M. Najafabadi, Probing the Anomalous FCNC Interactions in Top-Higgs Final State and Charge Ratio Approach, Phys. Rev. D 89 (2014) 054011 [arXiv:1402.3073] [INSPIRE].

[29] B.F. Yang, N. Liu and J.Z. Han, Top Quark FCNC Decay to 125 GeV Higgs boson in the Littlest Higgs Model with T-parity, Phys. Rev. D 89 (2014) 034020 [arXiv:1308.4852] [INSPIRE].

[30] D. Atwood, S.K. Gupta and A. Soni, Constraining the flavor changing Higgs couplings to the top-quark at the LHC, JHEP 10 (2014) 057 [arXiv: 1305.2427] [INSPIRE].

[31] Y. Wang, F.P. Huang, C.S. Li, B.H. Li, D.Y. Shao and J. Wang, Constraints on flavor-changing neutral-current $H t q$ couplings from the signal of $t H$ associated production with QCD next-to-leading order accuracy at the LHC, Phys. Rev. D 86 (2012) 094014 [arXiv: 1208.2902] [INSPIRE].

[32] J.J. Cao, L. Wang, L. Wu and J.M. Yang, Top quark forward-backward asymmetry, FCNC decays and like-sign pair production as a joint probe of new physics, Phys. Rev. D 84 (2011) 074001 [arXiv: 1101.4456] [INSPIRE].

[33] J.J. Cao, C.C. Han, L. Wu, J.M. Yang and M.C. Zhang, SUSY induced top quark FCNC decay $t \rightarrow$ ch after Run I of LHC, Eur. Phys. J. C 74 (2014) 3058 [arXiv:1404.1241] [INSPIRE].

[34] J.J. Cao et al., SUSY-induced FCNC top-quark processes at the large hadron collider, Phys. Rev. D 75 (2007) 075021 [hep-ph/0702264] [INSPIRE].

[35] H.-C. Cheng and I. Low, TeV symmetry and the little hierarchy problem, JHEP 09 (2003) 051 [hep-ph/0308199] [INSPIRE]. 
[36] H.-C. Cheng and I. Low, Little hierarchy, little Higgses and a little symmetry, JHEP 08 (2004) 061 [hep-ph/0405243] [INSPIRE].

[37] I. Low, T parity and the littlest Higgs, JHEP 10 (2004) 067 [hep-ph/0409025] [InSPIRE].

[38] J. Hubisz and P. Meade, Phenomenology of the littlest Higgs with T-parity, Phys. Rev. D 71 (2005) 035016 [hep-ph/0411264] [INSPIRE].

[39] S. Yang, Associated production of the Higgs boson and a single top quark in the littlest Higgs model at Large Hadron Collier, Phys. Lett. B 675 (2009) 352 [arXiv:0904.1646] [INSPIRE].

[40] C.-R. Chen, K. Tobe and C.-P. Yuan, Higgs boson production and decay in little Higgs models with T-parity, Phys. Lett. B 640 (2006) 263 [hep-ph/0602211] [INSPIRE].

[41] J. Alwall, M. Herquet, F. Maltoni, O. Mattelaer and T. Stelzer, MadGraph 5: Going Beyond, JHEP 06 (2011) 128 [arXiv:1106.0522] [INSPIRE].

[42] A. Belyaev, N.D. Christensen and A. Pukhov, CalcHEP 3.4 for collider physics within and beyond the Standard Model, Comput. Phys. Commun. 184 (2013) 1729 [arXiv:1207.6082] [INSPIRE].

[43] A. Belyaev, C.-R. Chen, K. Tobe and C.-P. Yuan, Phenomenology of littlest Higgs model with T-parity: including effects of T-odd fermions, Phys. Rev. D 74 (2006) 115020 [hep-ph/0609179] [INSPIRE].

[44] J. Pumplin, D.R. Stump, J. Huston, H.L. Lai, P.M. Nadolsky and W.K. Tung, New generation of parton distributions with uncertainties from global QCD analysis, JHEP 07 (2002) 012 [hep-ph/0201195] [INSPIRE].

[45] Particle Data Group collaboration, K.A. Olive et al., Review of Particle Physics, Chin. Phys. C 38 (2014) 090001 [inSPIRE].

[46] J. Reuter, M. Tonini and M. de Vries, Littlest Higgs with T-parity: Status and Prospects, JHEP 02 (2014) 053 [arXiv: 1310.2918] [INSPIRE].

[47] J. Hubisz, P. Meade, A. Noble and M. Perelstein, Electroweak precision constraints on the littlest Higgs model with T parity, JHEP 01 (2006) 135 [hep-ph/0506042] [INSPIRE].

[48] B.F. Yang, X.L. Wang and J.Z. Han, The Study of the contribution of the LHT model to Z $b \bar{b}$ coupling, Nucl. Phys. B 847 (2011) 1 [arXiv:1103.2506] [INSPIRE].

[49] B.F. Yang, G. Mi and N. Liu, Higgs couplings and Naturalness in the littlest Higgs model with T-parity at the LHC and TLEP, JHEP 10 (2014) 047 [arXiv: 1407.6123] [INSPIRE].

[50] L. Wang, J.M. Yang and J.Y. Zhu, Dark matter in the little Higgs model under current experimental constraints from the LHC, Planck and Xenon data, Phys. Rev. D 88 (2013) 075018 [arXiv: 1307.7780 ] [INSPIRE].

[51] C.-R. Chen, M.-C. Lee and H.-C. Tsai, Implications of the Little Higgs Dark Matter and T-odd Fermions, JHEP 06 (2014) 074 [arXiv: 1402.6815] [INSPIRE].

[52] E. Conte, B. Fuks and G. Serret, MadAnalysis 5, a user-friendly framework for collider phenomenology, Comput. Phys. Commun. 184 (2013) 222 [arXiv:1206.1599] [INSPIRE].

[53] CMS collaboration, Identification of b-quark jets with the CMS experiment, 2013 JINST 8 P04013 [arXiv: 1211.4462] [INSPIRE].

[54] J. Chang, K.M. Cheung, J.S. Lee and C.-T. Lu, Probing the Top-Yukawa Coupling in Associated Higgs production with a Single Top Quark, JHEP 05 (2014) 062 [arXiv: 1403.2053] [INSPIRE]. 